BONPLANDIA 19(1): 91-96. 2010

\title{
LAS ILUSTRACIONES DE LA «HISTORIA GENERAL Y NATURAL DE LAS INDIAS, ISLAS Y TIERRAFIRME DEL MAR OCÉANO» DE GONZALO FERNANDEZ DE OVIEDO Y VALDÉZ
}

\author{
ANTONIO KRAPOVICKAS ${ }^{1}$
}

\begin{abstract}
Summary: Krapovickas, A. 2010. The illustrations of the «Historia General y Natural de las Indias, Islas y Tierrafirme del Mar Océano» of Gonzalo Fernández de Oviedo y Valdéz. Bonplandia 19(1): 91-96. ISSN: 0524-0476.

The Historia General y Natural de las Indias by Oviedo published in 1535 has original illustrations that in the edition realized by Amador de los Ríos in 1851-1855 some of them were changed and other ones incorporated. In some cases the errors are huge like the illustration of a weasel with an Australian kangaroo. These errors disqualify all the changes realized by Amador de los Ríos in the 1851-1855 edition. For this reason, are presented here all the original illustrations of the first edition of the natural history.
\end{abstract}

Key words: natural history, original illustrations.

Resumen: Krapovickas, A. 2010. Las ilustraciones de la «Historia General y Natural de las Indias, Islas y Tierrafirme del mar Océano de Gonzalo Fernández de Oviedo y Valdéz». Bonplandia 19(1): 91-96. ISSN: 0524-0476.

\begin{abstract}
La Historia General y Natural de las Indias de Oviedo publicada en 1535 tiene ilustraciones originales que en la edición de Amador de los Ríos de 1851-1855 algunas fueron reemplazadas y se agregaron nuevas. En algunos casos los errores son garrafales como ilustrar a la comadreja con un canguro de Australia. Estos errores descalifican todos los agregados que Amador de los Ríos hizo a la edición de 1851-1855. Por ello, ahora se presentan todas las ilustraciones originales de la primera edición, referentes a la historia natural.
\end{abstract}

Palabras clave: historia natural, ilustraciones primigenias.

Gonzalo Fernández de Oviedo y Valdéz, con justicia llamado el primer naturalista de América, nació en Madrid en agosto de 1478, cuando los reyes católicos iniciaban su reinado, y murió el 26 de junio de 1557, en Valladolid, según Fernández de Navarrete y José Amador de los Ríos. Varios historiadores de Santo Domingo, afirman que falleció en la ciudad primada de América, donde, aseguran, existe su tumba, según consta en el libro anti- guo de la Real Contaduría de dicha ciudad.

Cuando muchacho entró a servir como mozo de cámara y compañero de estudios del príncipe heredero don Juan y como tal, estuvo presente en Barcelona cuando Colón, de vuelta de su primer viaje del descubrimiento, presentose ante los reyes católicos con «los primeros indios e algunas muestras de oro e muchos papagayos e otras cosas de las que acá estas gentes usaban». Este episodio deci-

${ }^{1}$ Instituto de Botánica del Nordeste, Casilla de Correo 209, 3400 Corrientes, Argentina. 
dió su vocación, proponiéndose ser el relator de los nuevos sucesos y de las cosas de las nuevas tierras recién descubiertas.

El primer libro de botánica impreso, basado en la observación directa, apareció en 1530, apenas 5 años antes de la publicación de la primera parte de la Historia General y Natural. Su autor fue Brunfels, quién identificó sus plantas de Estrasburgo con las plantas que Dioscórides estudió en Asia Menor en el primer siglo de nuestra era. A este libro de Brunfels siguieron los «herbarios figurados» de Bock en 1539 y de Fuchs en 1542.

Los libros de Oviedo que tratan sobre América son dos: el «Sumario» y la «Historia General y Natural».

El «Sumario de la Natural y General Historia de las Indias» fue publicado por primera vez en Toledo el 15 de febrero de 1526.

El Sumario no es un resumen o compendio de la Historia General, sino una obra diferente, con un plan distinto y escrito en otras circunstancias. Fue redactado durante su segundo viaje a España, a invitación del emperador Carlos V, quién deseaba ser informado sobre el mundo natural de sus nuevos dominios. Escribió esta obra de memoria, pues sus apuntes y notas para la redacción de su obra cumbre estaban en Santo Domingo, lugar de su residencia. No tiene ilustraciones.

La «Historia General y Natural de las Indias, Islas y Tierrafirme del Mar Océano» es mucho más importante, es el producto del trabajo de toda su vida. Publicó una primera parte en Sevilla en 1535 y de ésta se hizo una segunda edición en Salamanca, en 1547, con el título de «Coronica de las Indias» y al parecer sin cambios en el texto.

Consta esta primera parte de 20 libros, divididos en capítulos de longitud desigual y una epístola dedicatoria, contenidos en 186 folios (de dos carillas cada uno), más los índices o tablas.

De estos 20 libros, los siguientes están dedicados a las Ciencias Naturales:

«Libro 7, de la agricultura

8, que tracta de los árboles fructíferos

9 , que tracta de los árboles silvestres

10, de los árboles medicinales e de las plantas e de sus propiedades
11, el cual tracta de las hiervas e simientes que se truxeron de España a esta Isla Española

12, el cual tracta de los animales que en esta Isla Española se hallaron e quales se truxeron de España; e generalmente se han visto, e de que hay noticia en otras islas e en la Tierra Firme

13, el cual tracta de los animales de agua

14 , el cual tracta de las aves

15 , el cual tracta de los animales los insectos»

Acompañan al texto algunas ilustraciones de artefactos indígenas, plantas y animales.

Una vez publicada la primera edición en 1535, Oviedo se dedicó a ampliar su Historia y ya tenía casi lista para su publicación esta nueva edición aumentada, cuando lo sorprendió la muerte en 1557.

Durante un largo lapso se consideraron perdidos los valiosos manuscritos de Oviedo. Por largo tiempo los eruditos conocieron solamente partes fragmentarias de su magna obra.

Recién a mediados del XIX, gracias a las diligencias de don José Amador de los Ríos (1813-1878), de la Academia de la Historia de Madrid, se hizo la edición más completa de la Historia General. La hizo Amador de los Ríos sobre la base de la primera edición y a varios códices mutilados, pero que mutuamente se complementaban. Gracias a este trabajo de erudito, La Academia de la Historia de Madrid publicó una voluminosa edición compuesta de 4 grandes volúmenes, aparecidos entre 1851 y 1855, acompañados de un completo estudio sobre la vida y obras de Oviedo.

En 1944 se imprimió por segunda vez esta edición de Amador de los Ríos, en Asunción del Paraguay, por la editorial Guarania, en 14 tomos, con prólogo de J. Natalicio González.

Hay una gran diferencia en el contenido de estas dos ediciones, la de 1535 y la de 1851. La primera, en lo que respecta a las Ciencias Naturales, es el producto principal de su experiencia, adquirida durante sus viajes y trabajos en Tierra Firme (norte de Colombia y Venezuela, Panamá, algo de Nicaruaga y las Antillas).

En cambio preparó la segunda y tercera parte en su cómodo retiro de Santo Domingo donde se dedicó principalmente al estudio de los documentos que llegaban a sus manos 
como Cronista Oficial.

Muchas de las cosas que describió en su primera parte las debió conocer por haberlas visto. Aquí es donde reside la grandeza de Oviedo como historiador. Era un naturalista innato y con toda seguridad debía viajar con una libreta de apuntes, pues de otra manera no se explica la exactitud de muchas de sus observaciones.

De que era un naturalista instintivo lo podemos ver al correr de las páginas de este libro. Por ejemplo, al hablar de la palta dice: «Estos son unos árboles grandes y la hoja ancha y algo semejante a la del laurel, pero es mayor y más verde». La palta pertenece a la misma familia que el laurel, las Lauráceas y Oviedo hizo la comparación correcta.

La edición de 1851 repite toda la primera

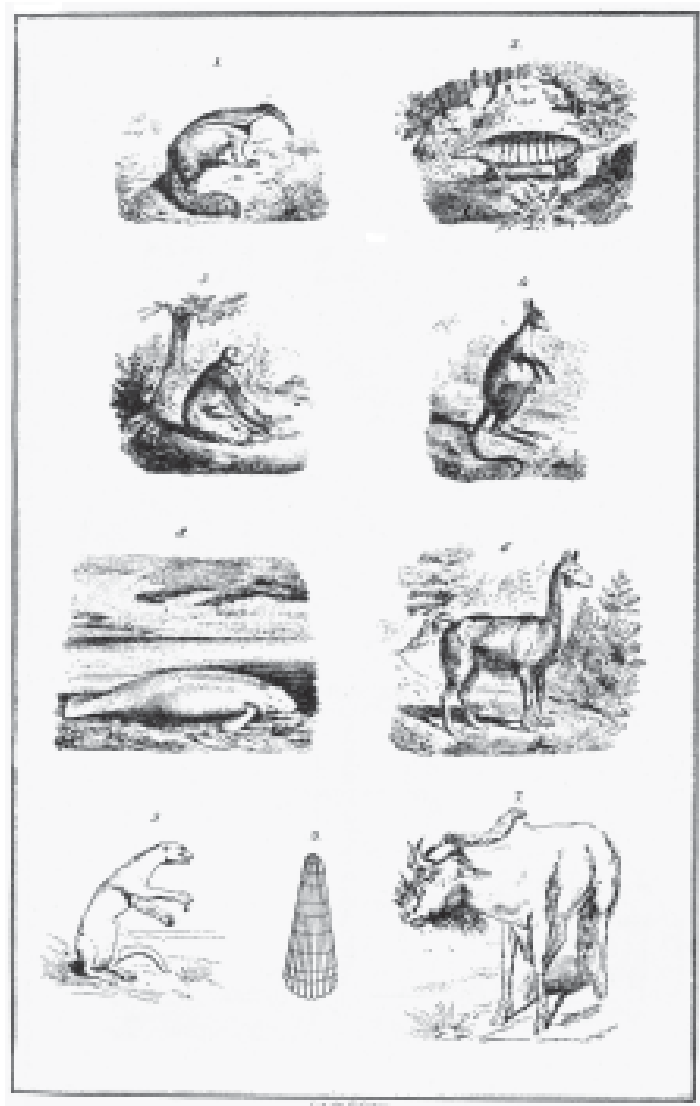

Fig. 1. Lámina de la edición de 1851 de la «Historia General y Natural de las Indias» de Oviedo donde la comadreja está representada por un canguro de Australia (4) y el búfalo americano por un búfalo africano (7). parte, pero cambia las ilustraciones. Reproduce las que figuran en la edición de 1535 y agrega algunas nuevas que no están en la primera edición.

Amador de los Ríos fue muy cuidadoso con los textos pero con las ilustraciones cometió algunos errores garrafales. Ilustró a la zarigüella o comadreja con un marsupial muy diferente: un canguro de Australia. El búfalo o bisonte con un búfalo de África (Fig. 1).

Estas inexactitudes inhabilitan el resto de las figuras que Amador de los Ríos incorpora en la edición de 1851. Por ello creo útil reproducir las ilustraciones auténticas, referidas a la historia natural que aparecieron en la primera edición de 1535. Para ello conté con un ejemplar depositado en la Biblioteca de la Universidad de Harvard (USA).
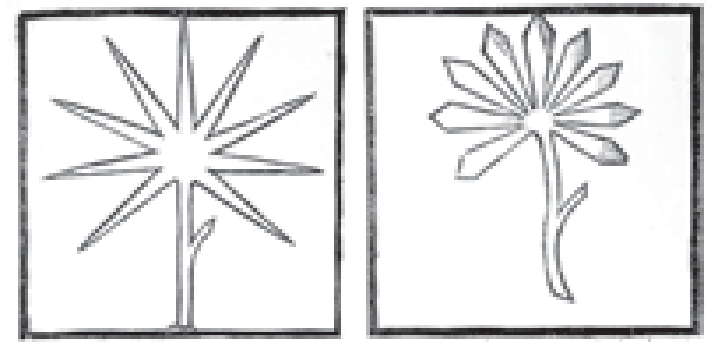

Fig. 2. Libro 7, capítulo 2, «del pan de los indios que se llama casabi» (Manihot utilissima).

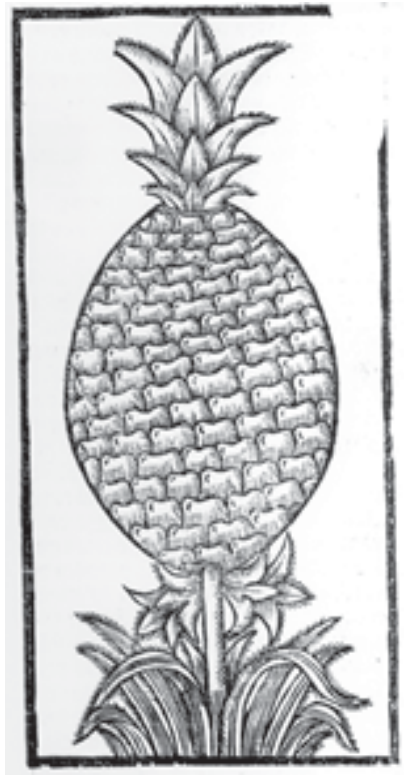

Fig. 3, Libro 7, capítulo 13, «de las piñas» (Ananas comosus). 


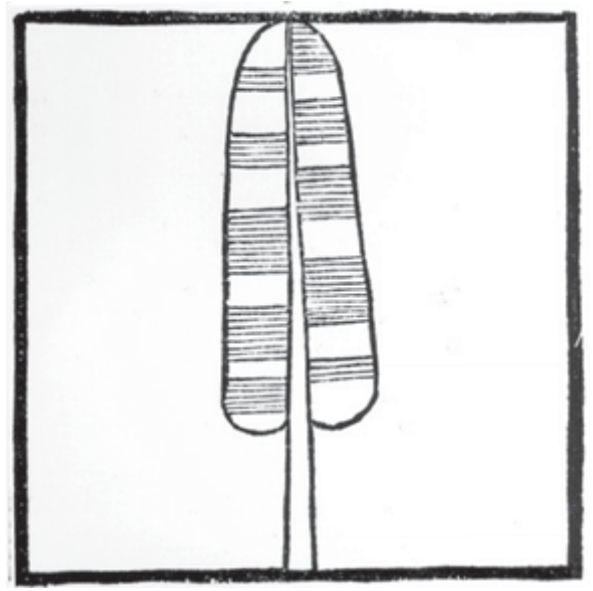

Fig. 4. Libro 8, capítulo 10 «hay una fruta que llaman plátanos».

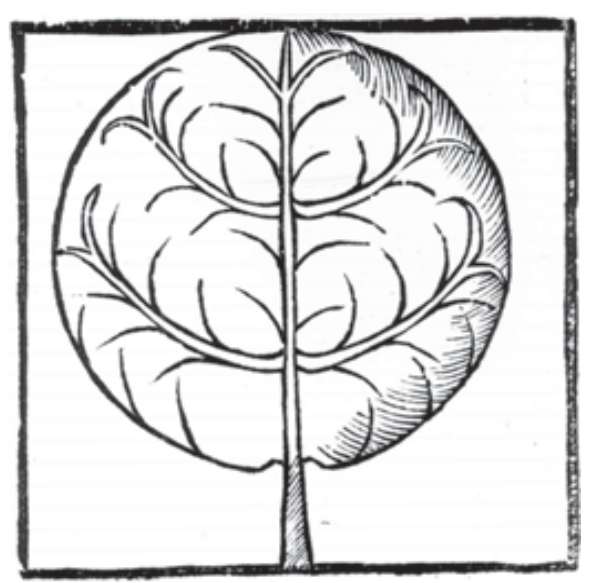

Fig. 6. Libro 8, capítulo 13, «del árbol llamado guiabara» (Coccoloba uvifera).

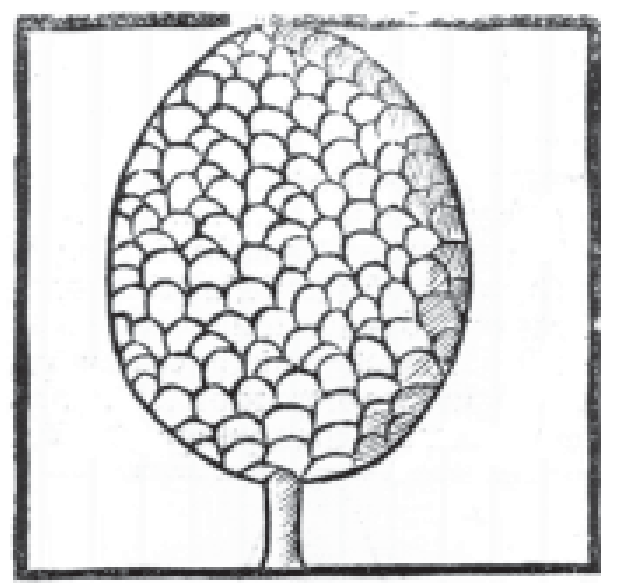

Fig. 8. Libro 8, capítulo 17, «del árbol guanábano e su fructa» (Annona muricata).

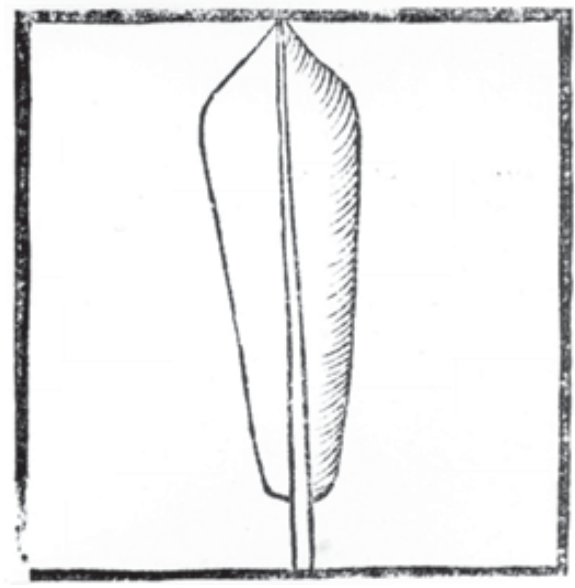

Fig. 5. Libro 8, capítulo 4, «del árbol llamado higüero».

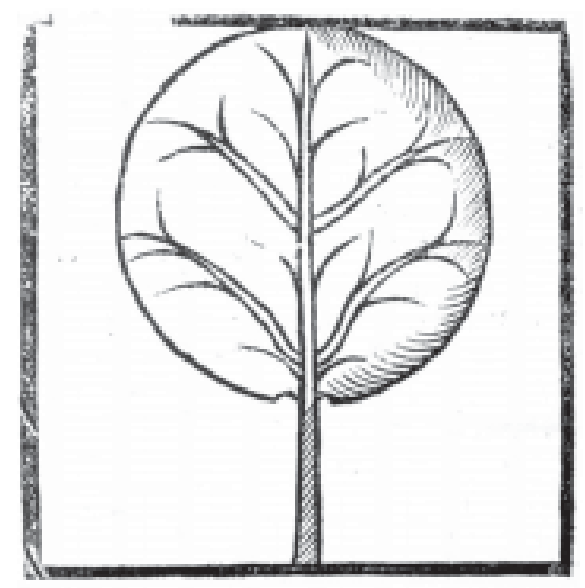

Fig.7. Libro 8, capítulo 14, «del árbol llamdo copey» (Clusia rosea).

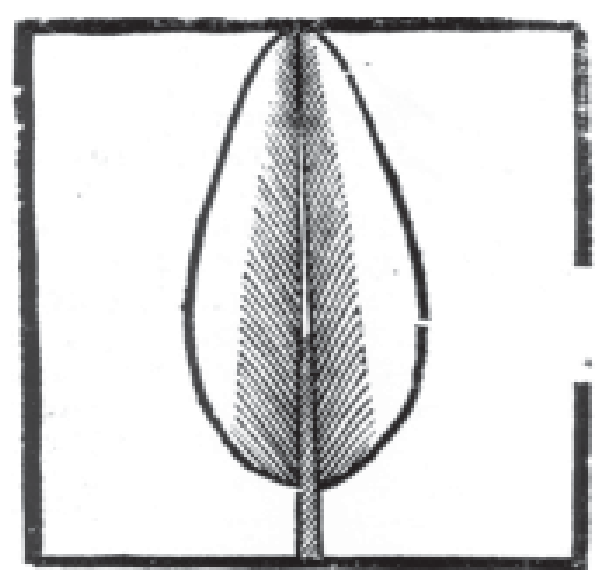

Fig. 9. Libro 8, capítulo 20, «el mamey» (Mammea americana). 


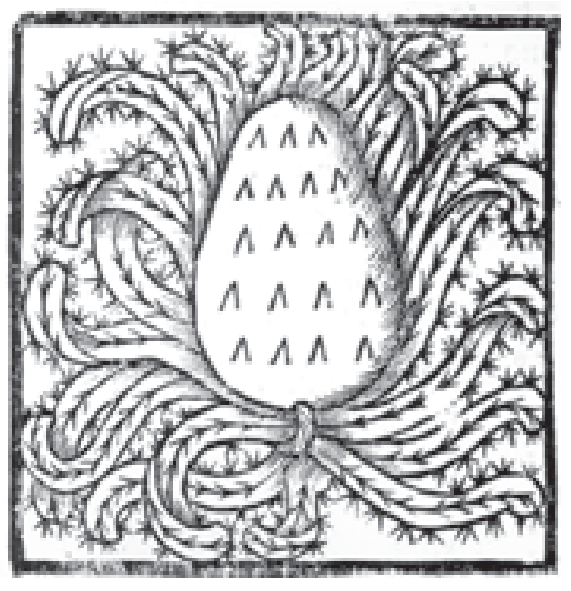

Fig. 10. Libro 8, capítulo 23, «de los cardones en que nace la fructa que llaman pitahaya» (dibujo realizado por Oviedo) (Harrisia nashii o Hylocereus triangularis).

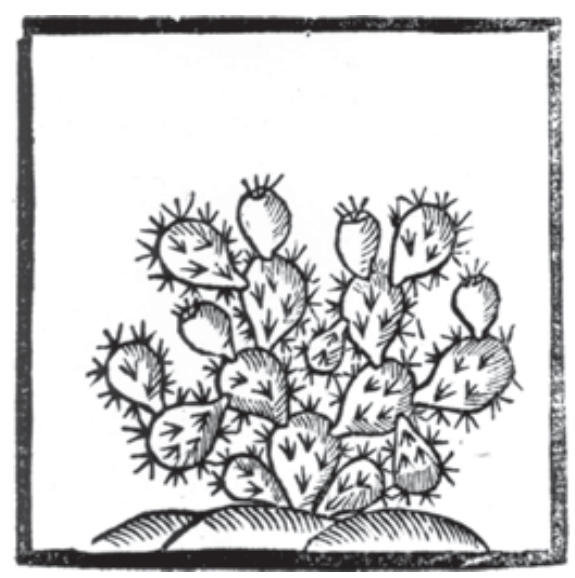

Fig. 12. Libro 8, capítulo 24,»de los cardos de las Tunas e su fructa».

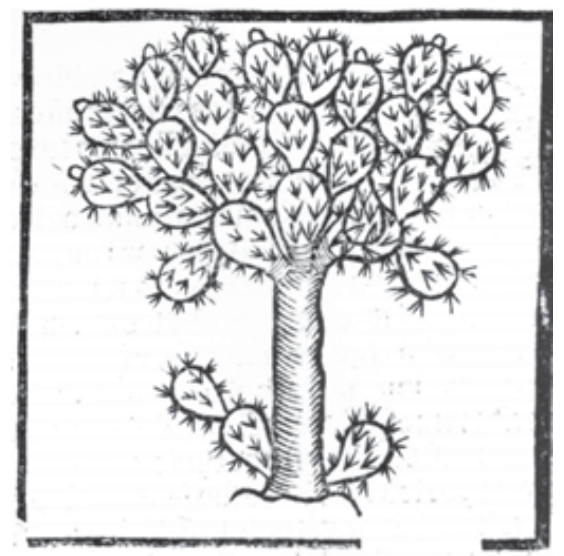

Fig. 13. Libro 10, capítulo 1, «del árbol e plancta con que se sueldan las quebraduras o cosas rompidas en la persona del hombre».

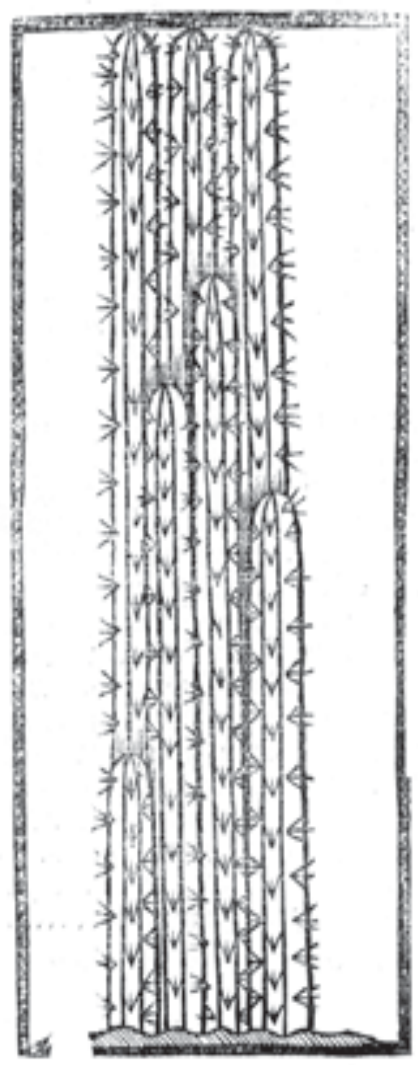

Fig. 11. Libro 8, capítulo 24, «de unos cardos altos y derechos...a los cuales llaman los cristianos lirios».

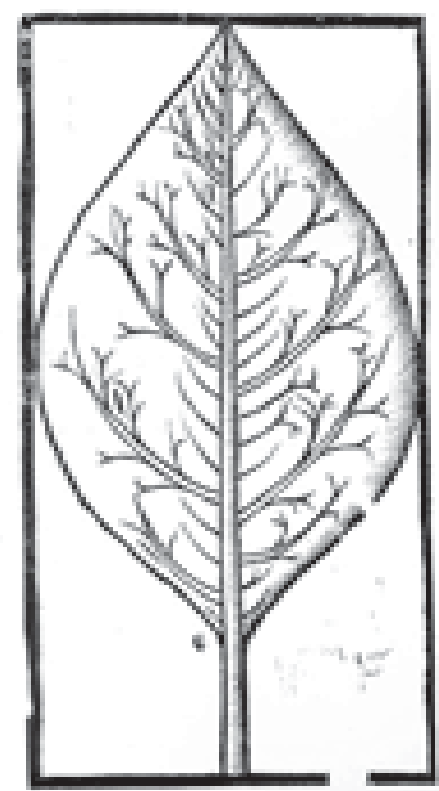

Fig. 14. Libro 11, capítulo 4, « de la yerba o plancta que los indios llaman goaconax y los cristianos le llaman bálsamo artificial o como les plaze nombrar este licor». 
BONPLANDIA 19(1). 2010

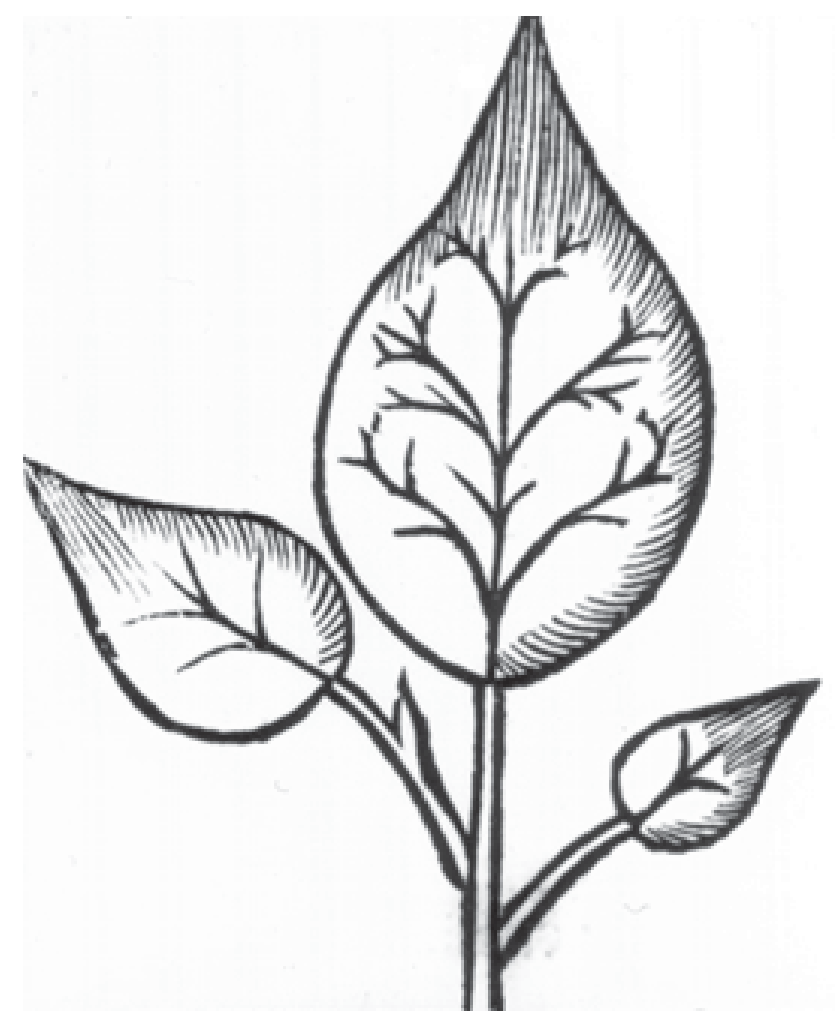

Fig. 15. Libro 11, capítulo 5, «de la yerba o plancta llamada perecebenuc»

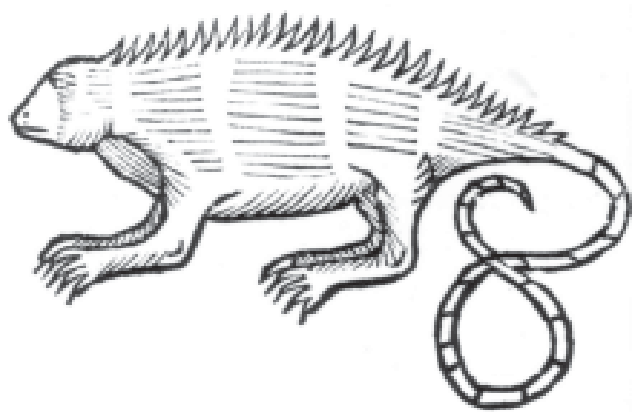

Fig. 16. Libro 13, capítulo 3, «de la yvana».

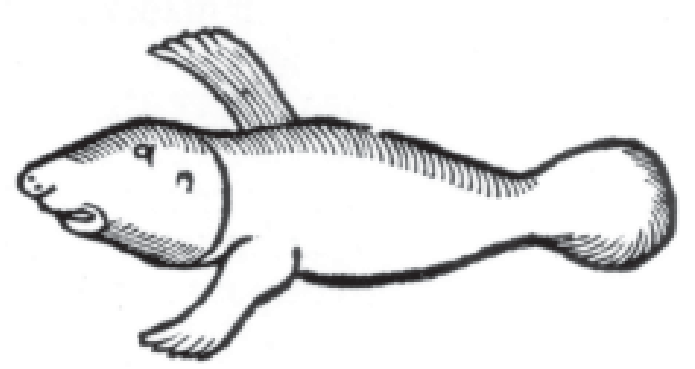

Fig. 17. Libro 13, capítulo 10, «del manatí». 